\title{
Performance of digital radiography with enhancement filters for the diagnosis of proximal caries
}

\section{Manuella Dias Furtado Belém ${ }^{(a)}$ Gláucia Maria Bovi Ambrosano(a) Cínthia Pereira Machado Tabchoury ${ }^{(b)}$ \\ Rívea Inês Ferreira-Santos(c) Francisco Haiter-Neto(a)}

\footnotetext{
(a) Department of Oral Diagnosis, Faculdade de Odontologia de Piracicaba - FOP, Univ Estadual de Campinas - UNICAMP, Piracicaba, SP, Brazil.

(b) Department of Physiological Science, Faculdade de Odontologia de Piracicaba - FOP, Univ Estadual de Campinas UNICAMP, Piracicaba, SP, Brazil.

(c)Department of Pediatric Dentistry and Orthodontics, School of Dentistry, Univ Cidade de São Paulo - UNICID, São Paulo, SP, Brazil.
}

Declaration of Interests: The authors certify that they have no commercial or associative interest that represents a conflict of interest in connection with the manuscript.

Corresponding Author: Manuella Dias Furtado Belém

E-mail: manubelem@yahoo.com.br

Submitted: Aug 27, 2012

Accepted for publication: Feb 19, 2013

Last revision: Mar 06, 2013

\begin{abstract}
Enhancement filters are potentially supposed to improve the diagnostic performance of digital images. Thus, the aim of this study was to compare the performance of digital radiography with and without enhancement filters for the detection of induced proximal caries lesions. The total sample consisted of 120 sound human teeth (40 premolars, 80 molars). Enamel subsurface demineralization was induced in one of the proximal surfaces of 60 teeth. Standardized radiographs of all teeth were acquired after the demineralization phase using the Digora-Optime ${ }^{\circledR}$ system. Four radiologists examined the digital radiographs and applied the following filters provided by the Digora ${ }^{\circledR}$ for Windows 2.6 package: Negative, Sharpen and both (Negative plus Sharpen). Validation of radiographic diagnosis was carried out by Knoop cross-sectional microhardness profiling on the proximal surfaces. Intraobserver agreement was estimated using Kappa statistics (k). Sensitivity, specificity and overall accuracy were compared using ANOVA/Tukey test $(\alpha=5 \%)$. Intraobserver agreement ranged from good to very good/optimal ( $\mathrm{k}: 0.65-0.83$ ). Although not statistically significant, the highest sensitivity $(0.68 \pm 0.22)$ and accuracy $(0.76 \pm 0.16)$ values were observed using the Sharpen filter as opposed to the Negative filter, which presented the lowest performance indices $(0.57 \pm 0.13$ and $0.70 \pm 0.10$, respectively). Specificity ranged from 0.84 to 0.85 , considering all imaging modalities ( $p>0.05)$. Insofar as the Sharpen filter had the highest performance indices, it may be considered a useful adjunct for detecting subtle proximal caries lesions.
\end{abstract}

Descriptors: Dental Caries; Dental Enamel; Radiographic Image Enhancement; Diagnosis.

\section{Introduction}

Radiography is a very suitable method of diagnosing proximal caries lesions, in addition to its relatively good availability and simple technical demands. ${ }^{1-11}$ The enhancement of brightness, contrast and edges carries the potential for increasing the diagnostic value of digital radiographs. ${ }^{12,13}$ Some authors have stated that filter enhancement may increase the diagnostic accuracy for detection of proximal caries lesions. ${ }^{14}$ On the other hand, no significant differences have been found between enhanced and original images acquired with $\mathrm{RVG}^{\circledR 10}$ and Vistascan ${ }^{\circledR 11}$ systems.

To date, there is but scant information on the performance of the 
Digora ${ }^{\circledR}$ Sharpen filter for the diagnosis of proximal caries lesions. In the Digora ${ }^{\circledR}$ for Windows 2.6 package, the Sharpen filter is a unique tool that allows non-linear filtering, which groups pixels in submatrix-evidencing high-contrast regions. In addition, the Sharpen filter may be applied simultaneously to other filters, such as the Negative filter. Thus, the aim of this study was to compare the performance of digital radiographic images without filter enhancement and with enhancement by the Digora ${ }^{\circledR}$ Sharpen, Negative, and combination of Negative and Sharpen filters, for the detection of subsurface proximal enamel demineralization. The null hypothesis stated no differences between filtered and non-filtered images.

\section{Methodology}

This study was approved by the Institutional Review Board (protocol \#148/2009) and is in agreement with the ethical principles for research with humans.

\section{Specimen preparation}

Sound human premolars $(\mathrm{N}=40)$ and third molars $(\mathrm{N}=80)$ were selected after extraction for orthodontic and surgical reasons. The root portion of each tooth was embedded in a rectangular block of utility wax. The crowns were coated with a fast-drying acid-resistant red varnish (Colorama Express $^{\circledR}$, Colorama/CEIL, São Paulo, Brazil), leaving a $7 \mathrm{~mm}^{2}$ circular window of exposed enamel on one of the proximal surfaces. Specimens were numbered and then randomly assigned to two groups of 60 specimens ( 1 - no demineralization, 2 - demineralization).

\section{Demineralization phase}

A buffer solution, $50 \%$ saturated in relation to dental enamel, was tested in pilot studies and used to induce subsurface demineralization. ${ }^{7}$ This demineralizing solution contained $0.05 \mathrm{M}$ acetate buffer, $\mathrm{pH} 4.8,1.12 \mathrm{mM}$ calcium, $0.77 \mathrm{mM}$ phosphate, and $0.03 \mathrm{ppm}$ fluoride. ${ }^{15}$ The ratio recommended for use is $2 \mathrm{~mL}$ of demineralizing solution to $1 \mathrm{~mm}^{2}$ of exposed enamel. Since the exposed enamel area was $7 \mathrm{~mm}^{2}$, the teeth in group 2 were kept individually immersed in $14 \mathrm{~mL}$ of the demineralizing solution and incubated at $37^{\circ} \mathrm{C}$ for 120 days. After 60 days of immersion, the demineralizing solution was replaced to avoid supersaturation. ${ }^{7}$

To test the possibility of demineralizing solution penetration and ionic exchange after coating the tooth crown with the fast-drying acid-resistant red varnish, the specimens in group 1 were separated into two subgroups. Thirty specimens were kept individually in plastic recipients on a pellet of cotton moistened with distilled and deionized water, at $37^{\circ} \mathrm{C}$ for 120 days. The other 30 specimens were coated totally on their crown with the red nail varnish, and were kept immersed in demineralizing solution for 120 days at $37^{\circ} \mathrm{C}$. No significant difference was found between mean Knoop microhardness numbers (KHN) obtained for both subgroups $(\mathrm{p}>0.05)$. Hence, these subgroups were considered the control group, for statistical purposes.

\section{Image acquisition}

Standardized radiographs of all the specimens were taken after 120 days using the Digora-Optime ${ }^{\circledR}$ system (Orion Corp.; Soredex ${ }^{\circledR}$, Helsinki, Finland). The specimens were radiographed using a GE $1000^{\circledR}$ X-ray unit (General Electric Co., Milwaukee, USA), operating at $65 \mathrm{kVp}, 10 \mathrm{~mA}, 2.5 \mathrm{~mm}$ total aluminum filtration, and at a $32 \mathrm{~cm}$ focus-receptor distance. The exposure time selected was $0.16 \mathrm{~s}$. An acrylic device was manufactured to hold the specimen, the X-ray beam indicator device and the image receptor in a reproducible relationship (Figure 1). A constant specimen-receptor distance of $2 \mathrm{~cm}$ was maintained in this acrylic device, and the X-ray tube vertical and horizontal angulations were set at $0^{\circ}$ and $90^{\circ}$, respectively. A $1.5 \mathrm{~cm}$ thick acrylic plate was positioned in front of the specimens to simulate soft tissues. The image receptors were scanned at a standard resolution of $397 \mathrm{dpi}$.

Digital radiographs were then exported in TIFF (tagged image file format), 8 bits, to Digora ${ }^{\circledR}$ for Windows 2.6 (Soredex, Tuusula, Finland). The Negative filter was applied and the modified images were recoded and stored in TIFF ( 8 bits). The matrix sizes of the original and the enhanced images were $480 \times$ 632 and $476 \times 632$, respectively. Next, the Sharpen 
Figure 1 - Acrylic device holding the X-ray beam indicator, specimen and image receptor in a standardized position.
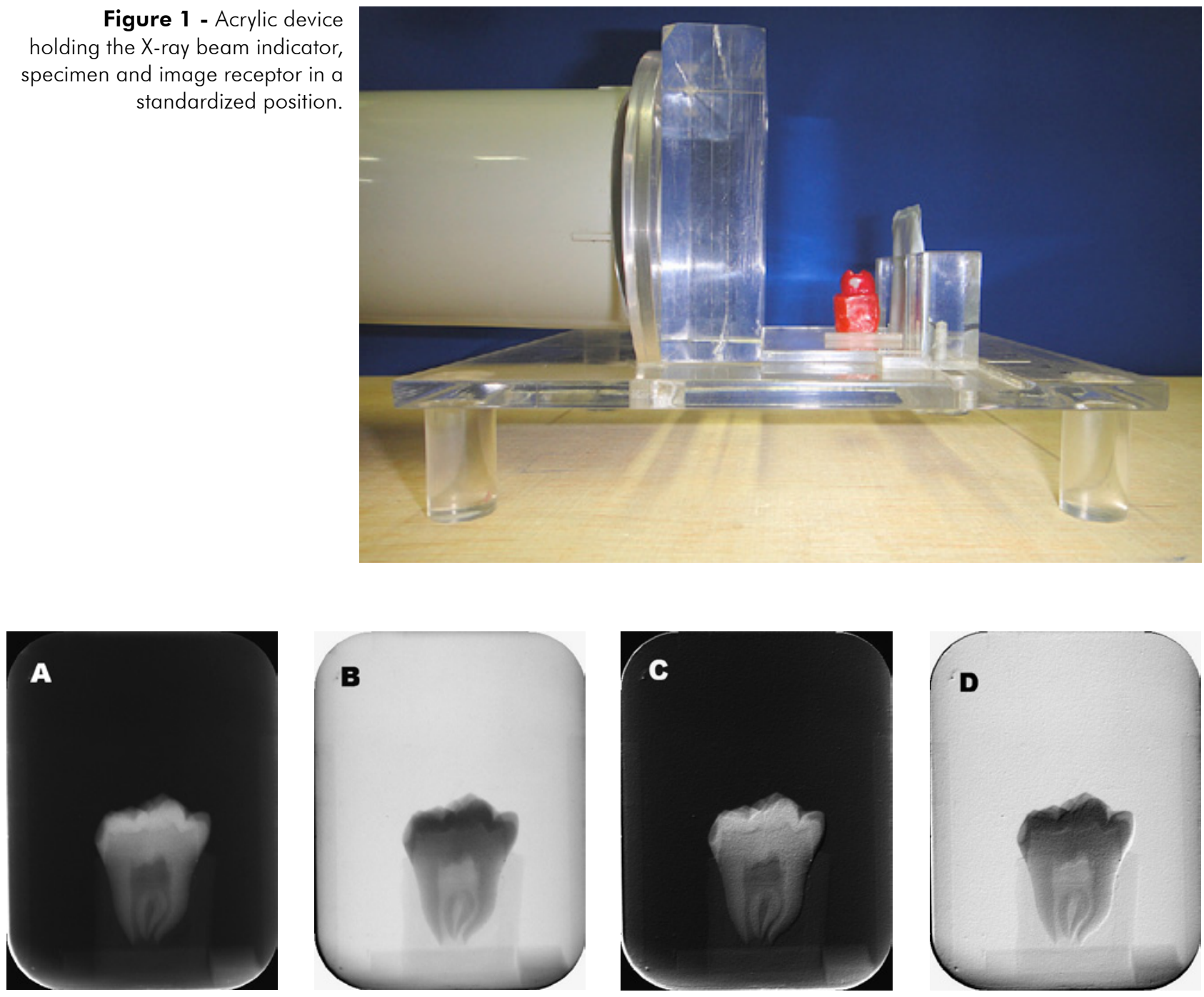

Figure 2 - Images of an experimental specimen showing subsurface demineralization. A: Without filter enhancement, B: Negative filter, C: Sharpen filter, and D: Negative plus Sharpen filters.

filter was applied and the enhanced images were recoded and stored in TIFF ( 8 bits) with the same matrix size. Both the Negative and the Sharpen filters were applied altogether, and these enhanced images were also recoded and stored in TIFF ( 8 bits). Four subsamples of images were generated: the Digora ${ }^{\circledR}$ original images and the images with filter enhancement: Negative, Sharpen and Negative plus Sharpen (Figure 2).

\section{Image assessment}

Four blinded and experienced dental radiologists assessed the digital radiographs using the Digora ${ }^{\circledR}$ for Windows 2.6 package. Following each calibra- tion session, the observers were instructed to analyze each image and score the proximal surfaces $(1=$ absence of subsurface demineralization, $2=$ presence of subsurface demineralization). Images were presented in random order on a 19-inch liquid crystal display monitor screen (W1952TQ, LG Electronics ${ }^{\circledR}$, Taubaté, Brazil), with a resolution of $1440 \times 900$ pixels, in true color (32 bits). All viewing was performed under uniform subdued lighting in a quiet and secluded room. ${ }^{8}$ The analog brightness and contrast controls on the monitor were kept constant during the assessments. The observers were positioned from 50 to $70 \mathrm{~cm}$ away from the monitor during image analysis. The duration of the 
interpretation sessions was not preset, but the observers were instructed to examine no more than 20 radiographs in each session to avoid visual fatigue.

\section{Reproducibility}

To estimate reproducibility, intraobserver agreement was analyzed using Kappa (k) statistics. Following the calibration session, the observers were asked to examine 20 images of the specimens twice. A ten-day interval was established between the first and second assessments. Intraobserver agreement ascertained $\mathrm{k}$ coefficients ranging from 0.65 to 0.83 , indicating good or very good/optimal reliability.

\section{Validation}

The enamel test areas were submitted to Knoop cross-sectional microhardness profiling, ${ }^{7}$ insofar as this method provides both microscopic visualization and measurement of mechanical resilience of the demineralized tissue, which in turn allows a quantitative assessment of the demineralization. ${ }^{15,16}$ This procedure was carried out using the FM Series ${ }^{\circledR}$ digital microhardness tester (Future-Tech Corp., Tokyo, Japan) connected to the FM-ARS ${ }^{\circledR} 7000$ software (Sun-Tec Corp., Novi, USA), which automatically calculated the KHN. ${ }^{17}$ One independent and

Table 1 - Overall true positive (TP), false positive (FP), true negative (TN), and false negative (FN) numbers for the detection of enamel subsurface demineralization by imaging modality.

\begin{tabular}{c|c|c|c|c}
\hline Imaging modality & TP & FP & TN & FN \\
\hline Original & 164 & 96 & 184 & 36 \\
\hline Negative & 149 & 111 & 188 & 32 \\
\hline Sharpen & 178 & 82 & 188 & 32 \\
\hline Negative \& Sharpen & 170 & 90 & 185 & 35 \\
\hline
\end{tabular}

well-trained operator carried out the microhardness measurements.

\section{Statistical analyses}

Cross-sectional microhardness profiling data were used as a reference for the true presence/absence of enamel subsurface demineralization. For this reason, absolute performance numbers (true positive, false positive, true negative and false negative) and indices-i.e. sensitivity (true positive ratio), specificity (true negative ratio) and overall accuracy (number of true positives + number of true negatives I all recordings)-were estimated for each imaging modality. In addition, receiver operating characteristic (ROC) analysis was used to assess the observers' performance in detecting proximal enamel subsurface demineralization. The areas under the ROC curves, designated as $A_{z}$, represent the efficiency of the diagnostic imaging modality. ANOVA/Tukey test $(\alpha=0.05)$ were used to compare the performance indices between the imaging modalities studied. All the analyses were carried out using the STATA ${ }^{\circledR} 7.0$ package (StataCorp. LP, College Station, USA).

\section{Results}

As summarized in Table 1, observers using the Sharpen filter made the highest number of true positive diagnoses, and also the smallest number of false positive observations.

Although not statistically significant, average sensitivity $(0.68 \pm 0.22)$ was higher for images enhanced with the Sharpen filter (Table 2). Mean overall accuracy was also higher for this imaging modality $(0.76 \pm 0.16)$, though not significant from a statistical standpoint. Conversely, the use of the Negative filter was associated to the lowest means of sensitivity and overall accuracy. Specificity ranged

Table 2 - Sensitivity, specificity and overall accuracy for the detection of subsurface demineralization by imaging modality (mean \pm SD).

\begin{tabular}{c|c|c|c}
\hline Imaging modality & Sensitivity & Specificity & Accuracy \\
\hline Original & $0.62 \pm 0.14(\mathrm{~A})$ & $0.84 \pm 0.07(\mathrm{~A})$ & $0.73 \pm 0.08(\mathrm{~A})$ \\
\hline Negative & $0.57 \pm 0.13(\mathrm{~A})$ & $0.85 \pm 0.02(\mathrm{~A})$ & $0.70 \pm 0.10(\mathrm{~A})$ \\
\hline Sharpen & $0.68 \pm 0.22(\mathrm{~A})$ & $0.85 \pm 0.06(\mathrm{~A})$ & $0.76 \pm 0.16(\mathrm{~A})$ \\
\hline Negative \& Sharpen & $0.65 \pm 0.17(\mathrm{~A})$ & $0.84 \pm 0.03(\mathrm{~A})$ & $0.74 \pm 0.14(\mathrm{~A})$ \\
\hline
\end{tabular}

Means followed by the same letter in the same column do not significantly differ according to the Tukey test at a $95 \%$ confidence interval 


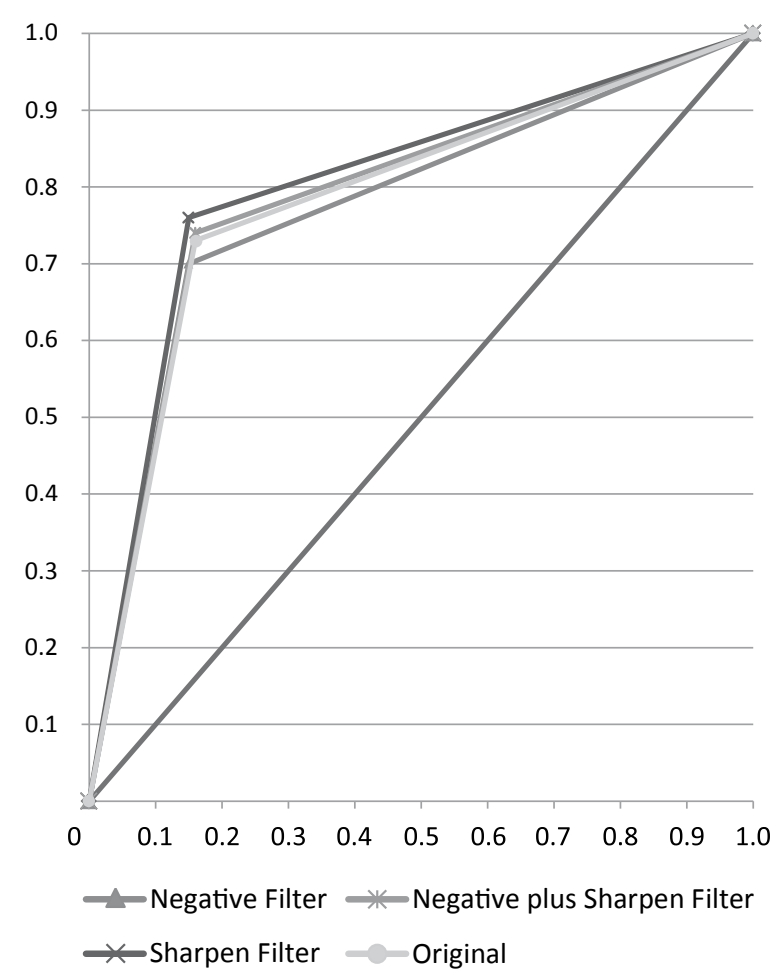

Figure 3 - Receiver operating characteristic curves for the imaging modalities. Original $\left(A_{z}=0.73\right)$, Negative filter $\left(A_{z}=0.70\right)$, Sharpen filter $\left(A_{z}=0.76\right)$ and Negative plus Sharpen filters $\left(A_{z}=0.74\right)$.

from 0.84 to 0.85 , considering all imaging modalities ( $p>0.05)$. It may be assumed, however, that the specificity mean values were generally high without affecting sensitivity $(0.62-0.68)$ for subsurface demineralization, except for the images with the Negative filter. The $\mathrm{A}_{z}$ was greater for the Sharpen filter, corroborating its higher overall accuracy (Figure 3).

\section{Discussion}

Proximal caries lesion depths are generally underestimated, but the measurements in this study were more accurate using the Digora ${ }^{\circledR}$ (photostimulable storage phosphor) and Dixi ${ }^{\circledR}$ (CCD-based) systems in comparison to other two digital intraoral systems. ${ }^{4}$ The Digora-Optime ${ }^{\circledR}$ system has more flexible plates, which are presumably more comfortable for the patients, and a reduced scanning time (from approximately 30 seconds to about 8 seconds) in relation to its predecessor Digora ${ }^{\circledR}$ FMX. However, an in vitro study on diagnostic performance for detecting non-cavitated proximal caries lesions reported that there was no statistically significant difference between the Digora ${ }^{\circledR}$ FMX and the Digora-Optime ${ }^{\circledR}$ (an older and a relatively newer version, respectively), in regard to overall accuracy. ${ }^{9}$ From a practical point of view, it may be suggested that both system versions are suitable for caries diagnosis.

Some studies mentioned that image enhancement with filters improved the detection of proximal caries lesions ${ }^{13,14}$ or reduced the observer variability. ${ }^{10}$ However, other authors suggested that filter enhancement did not provide a remarkable improvement in caries diagnosis accuracy in relation to the original digital and conventional radiographs. ${ }^{18}$ Because no statistically significant differences were found between the imaging modalities for the diagnosis of proximal subsurface demineralization (Table 2), the null hypothesis had to be accepted. This finding corroborates previous studies. ${ }^{10,18}$ At first glance, one should not indicate the use of filter enhancement for the diagnosis of subtle proximal demineralization. In fact, another experimental study also did not report any significant differences between the original images acquired with photostimulable storage phosphor plates and those processed with task-specific filters (Caries 1 and Caries 2). ${ }^{11}$ However, the use of the Fine enhancement filter was recommended whenever a dentist searches for a shallow caries lesion, even though this procedure did not provide significant statistical improvement in comparison with the original radiographs. ${ }^{11}$

The main objective of the enhancement techniques is to modify the physical attributes of an image to make it more suitable for a given task and a specific observer. ${ }^{18}$ Filters are tools derived from image processing algorithms (mathematical equations) included in imaging analyzing software applications. ${ }^{14}$ An enhancement filter could modify the input image (original image) to compensate for losses in image quality caused by underexposure or noise, ${ }^{11}$ rendering an output image (filtered image). The Sharpen filter included in the Digora ${ }^{\circledR}$ for Windows 2.6 package was developed with an algorithm that enables computer-assisted manipulation of the input image data array or matrix, grouping high-contrast pixels together in a submatrix, thus evidencing subtle changes in the output image. ${ }^{19}$ 
In general, radiographic examination provides low sensitivity and high specificity in the detection of non-cavitated caries lesions. ${ }^{20}$ This finding is in agreement with those presented in Table 2, albeit with the sensitivity and specificity observed for the Sharpen filter. As shown in Table 2, the DigoraOptime ${ }^{\circledR}$ radiographs enhanced with the Sharpen filter exhibited the most favorable relationship between sensitivity $(0.68)$ and specificity $(0.85)$, followed by the images with both Negative and Sharpen filters, and the original images (sensitivity: 0.62, specificity: 0.84 ). A plausible explanation for the superior performance of observers using the Sharpen filter may be related to the two-dimensional nature of the radiographic examination, which more frequently evidences the radiolucency of advanced lesions extending to the dentine. However, because the Sharpen filter can highlight contrast regions, proximal enamel subsurface demineralization that could have been overlooked in the original images may have been depicted in the filtered images.

The most unbalanced relationship was registered for the images with the Negative filter. Interestingly, the radiographs enhanced with both Negative and Sharpen filters had the second best performance indices (Table 2). Considering absolute numbers (Table 1) and indices (Table 2) for the Sharpen filtered images, in relation to the original and the Negative filter images, it may be assumed that the Sharpen filter actually improved the diagnostic performance of these two imaging modalities. Not surprisingly, the Negative filter yielded the lowest performance

\section{References}

1. Zhang ZL, Qu XM, Li G, Zhang ZY, Ma XC. The detection accuracies for proximal caries by cone-beam computerized tomography, film, and phosphor plates. Oral Surg Oral Med Oral Pathol Oral Radiol Endod. 2011 Jan;111(1):103-8.

2. Haiter-Neto F, Ferreira RI, Tabchoury CPM, Bóscolo FN. Linear and logarithmic subtraction for detecting enamel subsurface demineralization. Dentomaxillofac Radiol. 2005 May;34(3):133-9.

3. Seneadza V, Koob A, Kaltschmitt J, Staehle HJ, Duwenhoegger J, Eickholz P. Digital enhancement of radiographs for assessment of interproximal dental caries. Dentomaxillofac Radiol. 2008 Mar;37(3):142-8. indices for detecting proximal subsurface demineralization (Table 2). This result may be related to the radiographic interpretation method, since dentists are taught and trained to diagnose caries lesions as radiolucent images.

As in many other studies on the performance of imaging modalities for the detection of caries lesions, ${ }^{2,6,13,21,22}$ dental radiologists were selected as observers. These professionals are well-trained for imaging diagnosis; therefore, many enhancement tools may have little effect on their performance, as opposed to what would be expected from a general practitioner. Nevertheless, considering that caries lesion depth is often underestimated in radiographic images $^{4}$ and that proximal subsurface demineralization is difficult to be detected, ${ }^{2,6,11}$ a diagnostic adjunct that could add any improvement in the radiographic image with no additional cost or significant time-consuming techniques should be recommended for and welcomed by dental radiologists and general practitioners. Therefore, the applicability of the Sharpen filter to Digora-Optime ${ }^{\circledR}$ radiographs should be considered as a suitable adjunct for enhancing the diagnosis of proximal surface demineralization such as caries lesion.

\section{Conclusion}

The Digora-Optime ${ }^{\circledR}$ images with the Sharpen filter had the best performance-related indices. Therefore, the use of the Sharpen filter is advisable to improve the diagnosis of incipient proximal caries lesions.

4. Jacobsen JH, Hansen B, Wenzel A, Hintze H. Relationship between histological and radiographic caries lesion depth measured in images from four digital radiography systems. Caries Res. 2004 Jan-Feb;38(1):34-8.

5. Haiter-Neto F, Wenzel A, Gotfredsen E. Diagnostic accuracy of cone beam computed tomography scans compared with intraoral image modalities for detection of caries lesions. Dentomaxillofac Radiol. 2008 Jan;37(1):18-22.

6. Ferreira RI, Haiter-Neto F, Tabchoury CPM, Paiva GAN, Bóscolo FN. Assessment of enamel demineralization using conventional, digital, and digitized radiography. Braz Oral Res. 2006 Apr-Jun;20(2):114-9. 
7. Ferreira RI, Haiter-Neto F, Tabchoury CPM, Bóscolo FN. In vitro induction of enamel subsurface demineralization for evaluation of diagnostic imaging methods. J Appl Oral Sci. 2007 Oct;15(5):392-8.

8. Wenzel A, Haiter-Neto F, Gotfredsen E. Influence of spatial resolution and bit depth on detection of small caries lesions with digital receptors. Oral Surg Oral Med Oral Pathol Oral Radiol Endod. 2007 Mar;103(3):418-22.

9. Haiter-Neto F, Pontual AA, Frydenberg M, Wenzel A. A comparison of older and newer versions of intraoral digital radiography systems: diagnosing noncavitated proximal carious lesions. J Am Dent Assoc. 2007 Oct;138(10):1353-9.

10. Haiter-Neto F, Pontual AA, Frydenberg M, Wenzel A. Detection of non-cavitated approximal caries lesions in digital images from seven solid-state receptors with particular focus on task-specific enhancement filters. An ex vivo study in human teeth. Clin Oral Investig. 2008 Sep;12(3):217-23.

11. Haiter-Neto F, Casanova MS, Frydenberg M, Wenzel A. Taskspecific enhancement filters in storage phosphor images from the Vistascan system for detection of proximal caries lesions of known size. Oral Surg Oral Med Oral Pathol Oral Radiol Endod. 2009 Jan;107(1):116-21.

12. Kamburoğlu K, Murat S, Yüksel SP, Cebeci AR, Paksoy CS. Occlusal caries detection by using a cone-beam CT with different voxel resolutions and a digital intraoral sensor. Oral Surg Oral Med Oral Pathol Oral Radiol Endod. 2010 May;109(5):e63-9.

13. Møystad A, Svanæs DB, van der Stelt PF, Gröndahl H-G, Wenzel A, van Ginkel FC, et al. Comparison of standard and task-specific enhancement of Digora storage phosphor images for approximal caries diagnosis. Dentomaxillofac Radiol. 2003 Nov;32(6):390-6.
14. Lehmann TM, Troeltsch E, Spitzer K. Image processing and enhancement provided by commercial dental software programs. Dentomaxillofac Radiol. 2002 Jul;31(4):264-72.

15. Argenta R, Tabchoury C, Cury JA. A modified pH-cycling model to evaluate fluoride effect on enamel demineralization. Pesqui Odontol Bras. 2003 Jul-Sep;17(3):241-6.

16. Featherstone JDB, ten Cate JM, Shariati M, Arends J. Comparison of artificial caries-like lesions by quantitative microradiography and microhardness profiles. Caries Res. 1983;17(5):385-91.

17. Arends J, ten Bosch JJ. Demineralization and remineralization evaluation techniques. J Dent Res. 1992 Apr;71 Spec No:924-8.

18. Eickholz P, Kolb I, Lenhard M, Hassfeld S, Staehle H. Digital radiography of interproximal caries: effect of different filters. Caries Res. 1999 May-Jun;33(3):234-41.

19. Analoui M. Radiographic image enhancement. Part I: spatial domain techniques. Dentomaxillofac Radiol. 2001 Jan;30(1):1-9.

20. Bader JD, Shugars DA, Bonito AJ. A systematic review of the performance of methods for identifying carious lesions. J Public Health Dent. 2002 Fall;62(4):201-13.

21. Şenel B, Kamburoğlu K, Üçok Ö, Yüksel SP, Özen T, Avsever H. Diagnostic accuracy of different imaging modalities in detection of proximal caries. Dentomaxillofac Radiol. 2010 Dec;39(8):501-11.

22. Tsuchida R, Araki K, Okano T. Evaluation of a limited conebeam volumetric imaging system: comparison with film radiography in detecting incipient proximal caries. Oral Surg Oral Med Oral Pathol Oral Radiol Endod. 2007 Sep;104(3):412-6. 